\title{
"FEATURES OF APPLICATION OF AUTOPLAZMA FOR TREATMENT OF THE ODONTOGENNY INFECTION OF MAXILLOFACIAL AREA AT PATIENTS WITH DIABETES"
}

\author{
${ }^{1}$ Khamitova Firuza Artikovna, ${ }^{2}$ Safarova Mashhura Sulaymonovna \\ ${ }^{1}$ Assistant, Department of Surgeral Dentistry, Bukhara State Medical Institute, Bukhara, Uzbekistan. \\ ${ }^{2}$ Assistant, Department of Surgeral Dentistry, Bukhara State Medical Institute, Bukhara, Uzbekistan. \\ E-mail: dr.khamitova@mail.ru \\ E-mail: mashhura_ss@mail.ru
}

\begin{abstract}
:
The article is dedicated to the advanced multimodality therapy of the patients with odontogenic osteomyelitis of the jaws and its complications with local application of platelet enriched with plasma in the patients with diabetes mellitus taking into account the nature of the course of the inflammatory process. Platelet auto plasma is a highly active biological stimulator of regeneration processes owing to various growth factors contained in alpha granules of platelets, acting on all structural units of the surrounding tissue and stimulating regeneration processes. The article defines the use of platelet autoplasma and their advantage in the treatment of abscesses and phlegmon of the maxillofacial area in patients with diabetes mellitus.
\end{abstract}

Keywords:

phlegmon, osteomyelitis, autoplasma, immunity, diabetes mellitus, antibiotic, growth factor, infiltrate, glycemia, pus, infection, detoxification.

Article Received: 18 October 2020, Revised: 3 November 2020, Accepted: 24 December 2020

\section{INTRODUCTION}

The frequent development of abscesses and phlegmon in the head and neck is due to the high prevalence of chronic focal odontogenic, tonsilogenic infection, as well as infectious and inflammatory lesions of the skin and oral mucosa. The most common form of septic inflammation is odontogenic suppurative inflammatory disease. The combination of odontogenic infection with diabetes forms a vicious circle in which the infection negatively affects metabolic processes, exacerbating insulin deficiency and increasing acidosis, and metabolic and microcirculatory disorders worsen the course of reparative processes in the lesion. Due to the reduced resistance of diabetes patients to infection, wounds predominate in necrotic processes, the classic symptoms that characterize inflammation are sometimes mild, and some may be absent. Suppurative processes can acquire a dystrophic nature, which is based on tissue acidosis. Quite often, in these patients there is a reactive course of the purulent process or a sluggish, protracted course of a wound infection after the removal of acute phenomena[1,2].

Currently, in the treatment of acute odontogenic purulent-inflammatory diseases of the maxillofacial region, a variety of methods are used, aimed both at the local focus of inflammation, and at the microorganism as a whole. A very important link in the treatment of inflammatory diseases of the maxillofacial region can be methods of local stimulation of soft tissue regeneration processes.

Platelet autoplasma is a highly active biological stimulator of regeneration processes due to various growth factors contained in alpha granules of platelets, acting on all structural units of the surrounding tissue and stimulating regeneration processes.

Purpose of work. To evaluate the effectiveness of the use of PRP-therapy in patients for the treatment of odontogenic inflammatory diseases of the MND. 


\section{MATERIALS AND METHODS}

In the clinic of maxillofacial surgery of the Bukhara regional multidisciplinary medical center in the period from 2015 to 2019 . there were 153 patients aged 17 to 70 years with odontogenic purulent-inflammatory diseases of the maxillofacial region against the background of diabetes. Among them were 64 men and 89 women. The duration of diabetes mellitus ranged from 3 to 18 years. In 70 patients, insulindependent diabetes was established, in 83 - noninsulin-dependent diabetes. Odontogenic phlegmon was diagnosed in 93 patients, an abscess in 24 patients, odontogenic osteomyelitis in 15 patients, odontic sinusitis in 21 patients

\section{RESULTS AND DISCUSSION}

In the general structure of patients with purulentinflammatory diseases of the maxillofacial region against the background of diabetes, more than half $(51.6 \%)$ of the patients observed moderate and severe course of the disease. All these patients were over 50 years old. In most of them, the course of the underlying disease was aggravated, in addition to diabetes mellitus, by two or three concomitant diseases: $38 \%$ suffered from hypertension, $47.8 \%$ from angina pectoris and atherosclerosis, $40.5 \%$ from obesity. Most patients were admitted to hospital in the later stages from the onset of purulent surgical disease[1,2].

The purulent process in these patients creates the prerequisites for the destruction of endogenous and exogenous insulin, which leads to an increase in insulin deficiency and thus to the decompensation of diabetes. Patients with diabetes who have developed a purulent process, from the moment of admission to the hospital need full intensive treatment aimed at both compensating for pathological altered metabolic processes and eliminating the purulent focus. There is no doubt that the key link in the treatment of purulent-inflammatory diseases of the maxillofacial region is etiotropic antibacterial therapy. From a practical point of view, the main importance is attached to the correct choice of antibiotic. It must be remembered that patients with diabetes require a special approach. In addition, the use of autologous plasma eliminates the possibility of allergic reactions[1,3].

The technical result of the method is achieved through the use of a new technique, namely: venous blood is collected from a patient in a volume of 10-25 ml, depending on the amount of inflammation, then the blood is centrifuged with an acceleration of $1000 \mathrm{G}$ for 6 minutes, after centrifugation 3 are taken from the tube, $20 \mathrm{ml}$ of the obtained plasma, then the syringes with the plasma are placed in a thermostat and incubated to obtain a gel, after which the obtained gel is cooled in a sterile tray for 3 minutes, then, using the connector, the syringe with the obtained gel is introduced into the in iltrata. The autoplasma obtained by centrifugation contains high platelets, which means the following growth factors: IGF (insulin-like growth factor), PDGF (plateletderived growth factor), PDEGF (platelet-derived growth factor of endothelial cells), VEGF or PDAF (growth factor of vascular endothelium), EGF (epidermal growth factor), TGF- $\beta$ ("family" of transforming growth factor), - FGF (fibroblast growth factor).

\section{CONCLUSIONS}

Treatment of purulent wounds in the background of diabetes should be carried out with the participation of an endocrinologist under the control of criteria that objectively reflect the course of the wound process: cytological examination of wound prints, morphological examination of wound tissues, determination of tissue homeostasis in the lesion, quantitative and qualitative microbiological analysis. The use of the above methods, as well as the volume and direction of the therapy in each case, are the prerogative of the attending physician. Our study showed that treatment of odontogenic infection with an autothrombocyte mass makes it possible to stop a purulent lesion and shorten the healing 
time of a purulent wound due to the stimulating effect of platelet growth factors on bone and collagen metabolism, proliferation of vascular endothelial cells, and reduces the risk of complications.

\section{REFERENCES}

[1] Studying of influence of a preparation of baktreriofag on nonspecific factors of immunity at patients with generalized odontogen flegmons maxillo - facial region» Pulatova Sh.K., Z.K. Raximov, Kambarova Sh.A., Xamitova F.A. 2(26)2019 «Тиббиётда янги кун»

[2] «Experience in the treatment of patients with odontogenic jaw cysts» Rakhimov Zokir Kayimovich, Khamitova Firuza Artikovna, Kambarova Shakhnoza Alihuseynovna, Pulatova Shahzoda Karimovna, Safarova Mashhura Sulaymonovna European Sciences review Scientific journal № 11-12 2018 (November-December) Volume 2 ISSN 2310-5577

[3] «Presence of impacted teeth as a determining factor of unfavorable splits in 1256 sagittal-split osteotomies [seecomments]»Precious D.S., Lung K.E., Pynn B.R., Goodday R.H. (ENG; includes abstract) //Oral Surg Oral Med Oral Pathol Oral Radiol Endod. 1998 Apr. 85(4). - P 362-5.

[4] Bernadsky Yu .N. Fundamentals of maxillofacial surgery and surgical dentistry. M.: Medical literature, 2000.416 s.

[5] Buraeva I.S., Sarkisov A.Ya., Ivanyuta I.V. Plazmolifting in dentistry // In the collection: Actual issues of clinical dentistry Collection of scientific papers. 2016. S. 135-137.

[6] Krainov S.V., Mikhalchenko V.F., Popova A.N., Yakovlev

[7] A.T., Linchenko I.V. The effect of immunomodulatory
[8] therapy on the cellular composition of gingival fluid in elderly

[9] people suffering from periodontitis // Problems of Dentistry. -

[10] 2018. - T. 14. - No. 1. - S. 21-26.

(n)

